Preprint HU-TFT-94-6

6 April 1994

\title{
MULTIPLICITIES FOR LHC NUCLEAR COLLISIONS USING HERA STRUCTURE FUNCTIONS
}

\author{
K.J. Eskola ${ }^{1}$, K. Kajantie ${ }^{2}$ and P.V. Ruuskanen ${ }^{3}$
}

\begin{abstract}
We compute in QCD perturbation theory the transverse energy carried by gluons, quarks and antiquarks with $p_{T} \geq p_{0} \approx 2 \mathrm{GeV}$ in $\mathrm{Pb}+\mathrm{Pb}$ collisions at $\sqrt{s}=5500 A \mathrm{GeV}$ by using structure functions compatible with the small- $x$ increase observed at HERA. This gives a perturbative estimate for the energy and entropy density of the bulk system at times $\tau \sim 0.1 \mathrm{fm}$. The predicted initial gluon entropy density gives a lower limit of about $2200 \ldots 3400$ for the final charged multiplicity. Sources of further entropy increase are discussed.
\end{abstract}

1) Department of Physics, P.O.Box 9, 00014 University of Helsinki, Finland; kjeskola@ fltxa.helsinki.fi.

2) Department of Theoretical Physics, P.O.Box 9, 00014 University of Helsinki, Finland; kajantie@phcu.helsinki.fi.

3) Research Institute for Theoretical Physics, P.O.Box 9, 00014 University of Helsinki, Finland; vruuskanen@phcu.helsinki.fi. 
The new ep-data from HERA [1-2] imply an increase in the structure function $F_{2}^{p}$ at small $x$ relative to the behaviour implied by earlier data. Distribution function analyses using the new data have been carried out [3-4]. These analyses, in particular, constrain the gluon distribution function, which is not directly measured.

The purpose of this letter is to estimate, using these new gluon distribution functions, the average multiplicity in a central $\mathrm{Pb}+\mathrm{Pb}$ collision at Large Hadron Collider (LHC) energies, $\sqrt{s}=5500 \mathrm{AGeV}$. As the first stage, we compute the transverse energy carried by minijets, gluons, quarks and antiquarks, with $p_{T} \geq p_{0} \approx 2 \mathrm{GeV}$, produced in these collisions [5]. This calculation is as reliable as calculations in lowest order perturbative QCD at a scale $2 \mathrm{GeV}$ are: the main uncertainties are higher order corrections, which we account for with a $K$ factor, and shadowing, which we neglect. The second stage is more model dependent. We assume that the produced gluons form a system in spacetime at $\tau_{i}=1 / p_{0}=0.1 \mathrm{fm}$ and calculate its energy density from $\epsilon\left(\tau_{i}\right)=\left\langle E_{T}^{A}(|y|<\right.$ $0.5)\rangle \times p_{0} /\left(\pi R_{A}^{2}\right)$ and estimate from this the entropy density. Assuming that the further expansion of the system is adiabatic, we obtain from this a lower limit for the total multiplicity: later dynamical phenomena can only increase the entropy.

The expected charged multiplicity is a crucial variable for the planning of experiments [6] and the theoretical predictions lie roughly in the range 1500-8000. Our result with the new gluon distributions [3-4] enchanced at small $x$ is $d N_{c h} / d y>2200$ for $x g(x,) \sim x^{-0.3}$ and $>3400$ for $x g(x) \sim x^{-0.5}$; for the old distributions [7], used in ref. [5], with $x g\left(x, 4 \mathrm{GeV}^{2}\right) \sim$ const, the limit is only $d N_{c h} / d y>910$. Note that these estimates assume a $K$ factor of 2 and do not take into account shadowing. A reliable error estimate would require the study of higher order corrections [8] and gluon shadowing [9] for each structure function set separately. These tasks will not be pursued here. However, some insight on the uncertainty is provided by the dependence of our results on different structure function sets and on the parameter $p_{0}$. In any case, the new HERA distribution functions have a very interesting impact on the LHC heavy ion experiment.

Proceeding to the computation, all details and the physical motivation are given in [5]. Given the distribution functions $f_{i / p}$, the basic quantities calculated are the integrated 
jet cross section

$$
\sigma_{\text {jet }}\left(p_{0}\right)=\int_{p_{0}^{2}}^{s / 4} d p_{T}^{2} d y_{1} d y_{2} \frac{1}{2} \frac{d \sigma_{\text {jet }}}{d p_{T}^{2} d y_{1} d y_{2}},
$$

and the first $p_{T}$ moment

$$
\left\langle E_{T}\right\rangle \sigma_{\text {jet }}\left(p_{0}\right)=\int d p_{T} d y_{1} d y_{2} \frac{d \phi}{2 \pi} \frac{1}{2} \frac{d \sigma_{\text {jet }}}{d p_{T} d y_{1} d y_{2}}\left(\epsilon_{1}+\epsilon_{2}\right) p_{T},
$$

where $\epsilon_{1}=\epsilon\left(p_{T}, y_{1}\right)=1$ if the parton falls inside the region of our interest and $=0$ otherwise. Especially, we are going to consider the central rapidity region $|y| \leq 0.5$. The differential cross section is given by

$$
\frac{d \sigma_{\text {jet }}}{d p_{T}^{2} d y_{1} d y_{2}}=\sum_{\substack{i j k l=\\ q, \bar{q}, g}} x_{1} f_{i / p}\left(x_{1}, p_{T}^{2}\right) x_{2} f_{j / p}\left(x_{2}, p_{T}^{2}\right){\frac{d \hat{\sigma}^{i j \rightarrow k l}}{d \hat{t}}}
$$

and the distribution functions used are D0', H and D-' by MRS [3-4]. The behaviour of the gluon distributions of these sets at the scale $Q=p_{T}=2 \mathrm{GeV}$ is illustrated in Fig. 1 .

The results with $p_{0}=2 \mathrm{GeV}$ are given in Table 1 , and the $p_{0}$ dependence is studied in Figs. 2 and 3. The numbers of Table 1 are on the pp level, to convert them to numbers appropriate for central $A A$ collisions we also need the value of the overlap function $T_{A A}(\mathbf{b})$. We shall use $T_{\mathrm{PbPb}}(0)=32 / \mathrm{mb}$. The estimate for the gluonic contribution to initial $E_{T}$ in one unit of $y$ near $y=0$ is then

$$
\begin{array}{rlrl}
\left\langle E_{T}^{A}(|y|<0.5)\right\rangle & =T_{\mathrm{PbPb}}(0) \sigma_{\text {jet }}\left(p_{0}\right)\left\langle E_{T}\right\rangle \\
& =2700 \mathrm{GeV} & \text { DO } 1: x g(x) \sim \text { const } \\
& =4540 \mathrm{GeV} & & \text { D0' }: x g(x) \sim \text { const } \\
& =8640 \mathrm{GeV} & \mathrm{H}: & x g(x) \sim x^{-0.3} \\
& =15300 \mathrm{GeV} & \text { D-' }: x g(x) \sim x^{-0.5}
\end{array}
$$

The increase in $E_{T}$ associated with the small- $x$ increase is significant. However, this $E_{T}$ is the final $E_{T}$ only if no further interactions take place in the system. The largest decrease in $E_{T}$ is obtained if the system thermalises.

To obtain a lower limit for the final multiplicity we shall first assume that the system thermalises immediately after formation at $\tau_{i}=0.1 \mathrm{fm}$ and expands adiabatically thereafter. Since the entropy is conserved in the comoving frame, we can estimate the final 
multiplicity from the entropy at the initial time $\tau_{i}$. Aiming at a lower limit we shall only consider the dominant gluonic component, which, as seen from Table 1, is about $80 \%$ of the total. Then pressure, energy density and entropy density are $p=a T^{4}, \epsilon=3 a T^{4}$ and $s=4 a T^{3}$ with $a=16 \pi^{2} / 90=1.75$. Since the longitudinal size of the comoving volume is $\Delta z=\tau \Delta y$ we can estimate $\epsilon\left(\tau_{i}\right)=\left\langle E_{T}^{A}(|y|<0.5)\right\rangle p_{0} / \pi R_{A}^{2}$ and obtain for the charged final multiplicity

$$
\begin{aligned}
\frac{d N_{c h}}{d y} & \approx \frac{2}{3} \frac{1}{3.6} \frac{d S}{d y} \approx \frac{2}{3} \frac{1}{3.6} \pi R_{A}^{2} 4 a T_{i}^{3} \tau_{i} \\
& =\frac{2}{3} \frac{4}{3.6}\left[\frac{1}{27} \pi R_{A}^{2} a \tau_{i}\left\langle E_{T}^{A}(|y|<0.5)\right\rangle^{3}\right]^{1 / 4} \\
& =914 \quad \mathrm{DO} 1 \\
& =1350 \quad \mathrm{D} 0^{\prime} \\
& =2180 \quad \mathrm{H}, \\
& =3360 \quad \mathrm{D}-^{\prime}
\end{aligned}
$$

The assumption of fast initial thermalization seems reasonable when new HERA structure functions are used. In this case the gluonic subsystem is already initially close to chemical equilibrium, in the sense that the gluon number density is close to the thermal density obtained by assuming that all the initial energy density, $\epsilon\left(\tau_{i}\right)$, is thermalised. In other words, the average energy of the gluons is close to $\epsilon / n \approx 2.7 T$ as in thermal gas of bosons. The average energy per gluon within $|y|<0.5$ is obtained from Table 1 as $\sigma_{\text {jet }}\left\langle E_{T}(|y|<0.5)\right\rangle / 2 \sigma_{\text {jet }}$. The results for different structure functions are

$$
\begin{aligned}
& E / \text { gluon }=4.1 \mathrm{GeV} \quad 2.7 T_{i}=2.0 \mathrm{GeV} \quad \text { DO1 } \\
& =3.6 \mathrm{GeV} \quad=2.2 \mathrm{GeV} \quad \mathrm{DO}^{\prime}, \\
& =3.2 \mathrm{GeV} \quad=2.6 \mathrm{GeV} \quad \mathrm{H}, \\
& =2.9 \mathrm{GeV} \quad=3.0 \mathrm{GeV} \quad \mathrm{D}^{\prime} \text {. }
\end{aligned}
$$

For the distributions with $x g(x) \sim$ const the thermalisation of gluons requires a degradation of their average energy by collisions. With the HERA structure functions the secondary interactions are only needed for changing the directions of momenta to make the distribution uniform.

The lower limit of multiplicity was obtained by considering only the gluonic component and assuming immediate complete thermalisation among gluons. Then the work done 
against longitudinal expansion is maximal, much of the $E_{T}$ is shifted towards larger rapidities and $\epsilon$ decreases $\sim \tau^{-4 / 3}$. Maximal multiplicity is obtained if the viscosity is strong enough to balance the work done by the pressure [10]. In this case all $E_{T}$ remains in the interval $|y|<0.5$ and the multiplicity can be estimated by using $E_{T} /$ particle $\approx$ $0.5 \mathrm{GeV}$. In a realistic intermediate situation one has to consider, starting from the initial conditions in Table 1, the gluon, the quark and the antiquark subsystems and their interactions $[10,11]$ which tend to thermalize the system, first the gluons, then possibly the quarks and antiquarks. Kinetic and chemical equilibria are attained to various degrees. All this is associated with an increase in entropy and multiplicity, but quantitative estimates depend on the details of modeling.

In summary, we have considered the effects of the increase of gluon distributions in the small-x region as indicated by the new HERA data on the predictions of perturbative QCD - at the small scale of $2 \mathrm{GeV}$ - on the partonic subsystem produced in heavy ion collisions at LHC energies. With due reservation concerning possible shadowing effects [9], the HERA results give a solid reason for expecting rather large multiplicities and thus increase the likelihood of observing new gluon-quark plasma phenomena at LHC.

\section{Acknowledgements}

KK thanks F. Eisele for asking the question which lead to this investigation. 


\begin{tabular}{lrrrr} 
Set & $\sigma_{\text {jet }}$ & $g$ & $q$ & $\bar{q}$ \\
\hline DO1 & 130 & 101 & 18.8 & 10.0 \\
& 12.1 & 10.0 & 1.12 & 1.00 \\
& & & & \\
D0' & 233 & 183 & 31.9 & 18.5 \\
& 23.6 & 19.5 & 2.13 & 2.00 \\
& & & & \\
H & 512 & 401 & 70.5 & 40.5 \\
& 50.9 & 42.4 & 4.31 & 4.15 \\
& & & & \\
D- & 1039 & 833 & 137 & 68.9 \\
& 93.3 & 81.5 & 6.02 & 5.83
\end{tabular}

\begin{tabular}{lrrrr} 
Set & $\sigma_{\text {jet }}\left\langle E_{T}\right\rangle$ & $g$ & $q$ & $\bar{q}$ \\
\hline DO1 & 994 & 787 & 136 & 70.5 \\
& 100 & 84.5 & 8.29 & 7.41 \\
& & & & \\
D0' & 1570 & 1241 & 212 & 120 \\
& 170 & 142 & 14.5 & 13.6 \\
& & & & \\
H & 3100 & 2430 & 425 & 241 \\
& 322 & 270 & 26.7 & 25.5 \\
& & & & \\
D- & 5890 & 4720 & 782 & 392 \\
& 549 & 479 & 35.8 & 34.5
\end{tabular}

Table 1. Values of $\sigma_{\text {jet }}\left(p_{0}\right)$ and $\sigma_{\text {jet }}\left(p_{0}\right)\left\langle E_{T}\right\rangle$ in units of mb and mbGeV calculated for $p_{0}=2 \mathrm{GeV}$ and for three different MRS distribution functions with $x g\left(x, 4 \mathrm{GeV}^{2}\right) \sim$ const $\left(\mathrm{D}^{\prime}\right), \sim x^{-0.3}(\mathrm{H})$ and $\sim x^{-0.5}$ (D-') [3-4]. The results with Duke-Owens set 1 distributions (DO1) [7] are shown for comparison. An overall factor $K=2$ is included. Both the total as well as the contribution of gluons, quarks and antiquarks is given. For each set the upper numbers are for all $y$, the lower for $|y|<0.5$. 


\section{References}

[1] H1 collaboration, I. Abt et al., DESY report DESY 93-117 (August 1993).

[2] Zeus collaboration, M. Derrick et al., DESY report DESY 93-110 (August 1993).

[3] A.D. Martin, W.J. Stirling and R.G. Roberts, Phys. Lett. B306 (1993) 145.

[4] A.D. Martin, W.J. Stirling and R.G. Roberts, RAL preprint 93-077 (1993).

[5] K.J. Eskola, K. Kajantie and J. Lindfors, Nucl.Phys. B323 (1989) 37.

[6] A Letter of Intent for A Large Ion Collider Experiment (ALICE), CERN/LHCC/9316.

[7] D.W. Duke and J.F. Owens, Phys. Rev. D30 (1984) 49.

[8] S.D. Ellis, Z. Kunszt and D.E. Soper, Phys. Rev. D40 (1990) 2121.

[9] K.J. Eskola, J. Qiu and X.-N. Wang, Phys. Rev. Lett. 72 (1994) 36, K.J. Eskola, Nucl. Phys. B400 (1993) 240.

[10] K.J. Eskola and M. Gyulassy, Phys. Rev. C47 (1993) 2329.

[11] T.S. Biró, E. van Doorn, B. Müller, M.H. Thoma and X.-N. Wang, Phys. Rev. C48 (1993) 1275.

\section{Figure Captions}

Fig. 1. Gluon distribution functions MRS D0', D-, and $\mathrm{H}[3-4]$ at the scale $Q=2 \mathrm{GeV}$. The old gluon distribution of Duke-Owens set 1 (DO1) [7] is shown for comparison.

Fig. 2. The integrated jet cross section $\sigma_{\text {jet }}\left(p_{0}\right)$ computed from eq. (1) with the sets of distribution functions marked on the figure and for $\sqrt{s}=200$ and $5500 \mathrm{GeV}$.

Fig. 3. The first $p_{T}$ moment $\sigma_{\text {jet }}\left(p_{0}\right)\left\langle E_{T}\right\rangle$ from eq. (2). The curves are as in Fig. 2. 
This figure "fig1-1.png" is available in "png" format from: http://arxiv.org/ps/hep-ph/9404237v1 
This figure "fig1-2.png" is available in "png" format from: http://arxiv.org/ps/hep-ph/9404237v1 
This figure "fig1-3.png" is available in "png" format from: http://arxiv.org/ps/hep-ph/9404237v1 